\title{
Crescimento, nutrição e fixação biológica de nitrogênio em plantios mistos de eucalipto e leguminosas arbóreas
}

\author{
Selma Regina de Freitas Coelho(1), José Leonardo de Moraes Gonçalves(1), \\ Sergio Luis de Miranda Mello ${ }^{(1)}$, Rildo Moreira Moreira ${ }^{(1)}$, Eduardo Vinícius da Silva ${ }^{(1)}$ e Jean-Paul Laclau ${ }^{(2)}$ \\ (1)Escola Superior de Agricultura Luiz de Queiroz, Dep. de Ciências Florestais, Caixa Postal 9, CEP 13418-970 Piracicaba, SP. \\ E-mail: selmafcoelho@yahoo.com.br, jlmgonca@esalq.usp.br, sergio.luis.mello@itelefonica.com.br, moreira@carpa.ciagri.usp.br, \\ eduvini@igmail.com ${ }^{(2)}$ Cirad, Dep. Persyst, UPR 80, TA 10/D, 34398 Montpellier Cedex 5, França. E-mail: laclau@cirad.fr
}

\begin{abstract}
Resumo - O objetivo deste trabalho foi avaliar o crescimento inicial da parte aérea e do sistema radicular, a nutrição mineral e a fixação biológica de $\mathrm{N}_{2}(\mathrm{FBN})$ em plantios consorciados de Eucalyptus grandis e leguminosas arbóreas. O delineamento experimental foi o de blocos ao acaso com três repetições e sete tratamentos por bloco. Nas linhas de plantio, entre as plantas de E. grandis, foram plantadas, intercaladamente, leguminosas arbóreas nativas de matas brasileiras - Peltophorum dubium, Inga sp., Mimosa scabrella, Acacia polyphylla, Mimosa caesalpiniaefolia - e uma leguminosa exótica, Acacia mangium. Realizou-se, também, o plantio puro de E. grandis. Mimosa scabrella e A. mangium foram as leguminosas com maior crescimento. Eucalyptus grandis consorciado com $M$. scabrella cresceu menos, no entanto foi o povoamento com maior acumulação de biomassa. As densidades de raízes finas (DRF) do E. grandis foram 6 a 20 vezes maiores que as DRF das leguminosas na camada superficial do solo $(0-10 \mathrm{~cm}) 24$ meses após plantio. A DRF de M. scabrella e de M. caesalpiniaefolia foi maior na camada $30-50 \mathrm{~cm}$ e menor na camada $10-30 \mathrm{~cm}$. Os valores de $\delta^{15} \mathrm{~N}$ da $M$. scabrella indicam que $90 \%$ do $\mathrm{N}$ acumulado em seus tecidos é oriundo da FBN.

Termos para indexação: consórcio, raiz fina, plantio misto, leguminosa nativa.
\end{abstract}

\section{Growth, nutrition and biological fixation of nitrogen in mixed-species plantations of eucalypt with leguminous trees}

\begin{abstract}
The objective of this work was to assess interactions between species on the above and belowground growth, nitrogen uptake and biological nitrogen fixation (BNF) in mixed stands of Eucalyptus grandis and native leguminous $\mathrm{N}_{2}$-fixing trees. A complete randomized block design was installed with seven treatments and three blocks. Within the lines of the E. grandis seedlings, native leguminous $\mathrm{N}_{2}$-fixing trees - Peltophorum dubium, Inga sp., Mimosa scabrella, Acacia polyphylla, Mimosa caesalpiniaefolia - and one exotic leguminous plant, Acacia mangium, were intercropped. E. grandis was also solely planted. Mimosa scabrella and A. mangium were the legume trees that presented the highest growth. Although E. grandis showed a lower growth when combined with $M$. scabrella, this mixed-species stand exhibited the highest biomass accumulation. Eucalyptus grandis fine root densities (FRD) were 6-20 times higher than the FRD of the leguminous species in the upper soil layer $(0-10 \mathrm{~cm}) 24$ months after planting. The FRD of $M$. scabrella and $M$. caesalpiniaefolia in the $30-50 \mathrm{~cm}$ soil layer was higher than in the $10-30 \mathrm{~cm}$ layer. The $\delta^{15} \mathrm{~N}$ values of $M$. scabrella indicated that $90 \%$ of stocked $\mathrm{N}$ is derived from $\mathrm{BNF}$.
\end{abstract}

Index terms: consortium, fine root, mixed plantation, native leguminous.

\section{Introdução}

A grande procura por produtos florestais tem levado a um progressivo aumento da área florestal com povoamentos florestais monoespecíficos, de rápido crescimento, como os de eucaliptos, que apresentam alto potencial de exportação e depleção dos estoques de $\mathrm{N}$ do ecossistema.
Em áreas recém-florestadas com espécies de rápido crescimento, o estoque de $\mathrm{N}$ contido na matéria orgânica (MO) pode suprir a exigência das árvores por meio da mineralização do N orgânico (O'Connell et al., 1999). Nessas áreas, é comum pouca ou até mesmo a ausência de resposta das plantas a adições de fertilizantes nitrogenados (Barros et al., 1990). Entretanto, por meio do cultivo intensivo do solo, associado às rotações 
sucessivas de plantações florestais de rápido crescimento, como as de eucalipto, a depleção dos estoques de $\mathrm{N}$ do soloé rápida (O'Connell et al., 1999; Gonçalves et al., 2001).

Segundo estimativas de Gonçalves et al. (2001), alguns solos sob povoamentos homogêneos de Eucalyptus grandis no planalto ocidental paulista teriam reserva de $\mathrm{N}$ suficiente para atender às necessidades das árvores por três a cinco rotações (sete anos cada), se tornando, a partir de então, áreas potenciais de resposta à fertilização nitrogenada. Evidências desse efeito foram constatadas em plantações no Congo, após 50 anos de cultivo em solos de textura arenosa (Laclau et al., 2004). Quando o suprimento de N pela mineralização da $\mathrm{MO}$ e pelas deposições atmosféricas não são suficientes para atender às necessidades das árvores, adições suplementares de $\mathrm{N}$, por meio de fertilização mineral ou orgânica, esta última via fixação biológica de $\mathrm{N}_{2}(\mathrm{FBN})$ por leguminosas, podem constituir uma alternativa interessante (Binkley et al., 2000; Forrester et al., 2006).

Estudos com uso de leguminosas arbóreas em consórcio com espécies de eucalipto apresentam resultados distintos (Khanna, 1997; Bauhus et al., 2000; Balieiro et al., 2002; Forrester et al., 2006). Estes trabalhos evidenciam a atualidade do tema e sua importância na evolução tecnológica da silvicultura, tanto do ponto de vista econômico-social, como ambiental. A introdução de espécies leguminosas em povoamentos de eucaliptos nos solos de baixa fertilidade pode aumentar a capacidade de uso dos nutrientes uma vez que estes nutrientes são incorporados à biomassa e devolvidos ao solo via serapilheira, contribuindo com a manutenção ou restauração da fertilidade do solo (Li et al., 2001) e, por conseguinte, da produtividade florestal em longo prazo.

O objetivo deste trabalho foi avaliar o crescimento inicial da parte aérea e do sistema radicular, a nutrição mineral e a fixação biológica de $\mathrm{N}_{2}$ em plantios consorciados de Eucalyptus grandis e leguminosas arbóreas.

\section{Material e Métodos}

O trabalho foi realizado em condições de campo, na Estação Experimental de Ciências Florestais de Itatinga, pertencente à Escola Superior de Agricultura Luiz de Queiroz, Universidade de São Paulo, localizada em Itatinga, $23^{\circ} 0^{\prime} \mathrm{S}, 48^{\circ} 52^{\prime} \mathrm{O}$, altitude, $750 \mathrm{~m}$, SP. O clima local é mesotérmico úmido (Cwa), segundo a classificação de
Köppen. A precipitação média anual é de 1.360 mm, e nos meses mais secos, julho e agosto, a precipitação média mensal varia entre 30 e $60 \mathrm{~mm}$.

A temperatura média mínima é de $12,8^{\circ} \mathrm{C}$ nos meses mais frios, junho e julho, e a média anual é de $19,4^{\circ} \mathrm{C}$. A umidade relativa média anual é de $83,3 \%$. O solo da área foi caracterizado como Latossolo Vermelho-Amarelo (areia, $800 \mathrm{~g} \mathrm{~kg}^{-1}$; silte, $40 \mathrm{~g} \mathrm{~kg}^{-1}$ e argila, $160 \mathrm{~g} \mathrm{~kg}^{-1}$ ). Na camada de 0-20 cm, o pH em CaCl $20,01 \mathrm{~mol} \mathrm{~L}^{-1}$ é de 3,9, o teor de matéria orgânica é $20 \mathrm{~g} \mathrm{dm}^{-3}$, o teor de P-resina é $5 \mathrm{mg} \mathrm{dm}^{-3}$ e os teores de $\mathrm{K}, \mathrm{Ca}, \mathrm{Mg}$ e $\mathrm{Al}$ trocáveis iguais a $0,2,3,1$ e $13 \mathrm{mmol}_{\mathrm{c}} \mathrm{dm}^{-3}$, respectivamente. Nessas determinações químicas, foram usados os métodos descritos por Raij et al. (2001). Esse solo é um dos mais usados na eucaliptocultura no Estado de São Paulo. O relevo local é suavemente ondulado. A vegetação natural da região é o Cerrado.

A área experimental é florestada com Eucalyptus saligna há cerca de 60 anos, período em que foram realizadas cinco colheitas de madeira (corte raso), em rotações sucessivas do povoamento manejado no sistema de talhadia. Nas várias rotações, não foram realizadas adubações.

O solo foi preparado no sistema cultivo mínimo, realizando-se apenas uma subsolagem $(40 \mathrm{~cm}$ de profundidade) na linha de plantio. A fertilização básica de referência, aplicada em covetas laterais às mudas, foi de $100 \mathrm{~kg} \mathrm{ha}^{-1} \mathrm{de}_{2} \mathrm{O}_{5}$ (superfosfato triplo), $10 \mathrm{~kg} \mathrm{ha}^{-1} \mathrm{de}$ $\mathrm{K}_{2} \mathrm{O}$ (cloreto de potássio), $30 \mathrm{~kg} \mathrm{ha}^{-1}$ de FTE BR-12 (micronutrientes) e $2 \mathrm{Mg} \mathrm{ha}^{-1}$ de calcário dolomítico. Em cobertura, parcelados em duas aplicações iguais, aos três e oito meses pós-plantio, foram adicionados, no total, $150 \mathrm{~kg} \mathrm{ha}^{-1}$ de $\mathrm{K}_{2} \mathrm{O}$.

$\mathrm{O}$ experimento foi instalado em blocos ao acaso, com sete tratamentos e três repetições. Os tratamentos foram: T1: E. grandis consorciado com Peltophorum dubium (canafístula); T2: E. grandis consorciado com Inga sp. (ingá); T3: E. grandis consorciado com Mimosa scabrella (bracatinga); T4: E. grandis consorciado com Acacia polyphylla (monjoleiro); T5: E. grandis puro; T6: E. grandis consorciado com Mimosa caesalpiniaefolia (sansão-do-campo); T7: E. grandis consorciado com Acacia mangium (acácia). Cada parcela experimental possuía cem árvores e era formada por dez árvores na linha vs. dez árvores na entrelinha, com bordadura dupla. $\mathrm{O}$ espaçamento de plantio foi de $3 \mathrm{~m}$ por $3 \mathrm{~m}$ para o E. grandis puro e misto, com 1.111 árvores 
por hectare. As leguminosas foram plantadas na linha de plantio entre as plantas de E. grandis, em linhas alternadas, com 555 árvores por hectare. A densidade de leguminosa de $50 \%$ da densidade do E. grandis foi escolhida a partir dos resultados alcançados no mesmo local num experimento consorciando E. grandis com várias densidades de A. mangium (Bouillet et al., 2006).

$\mathrm{Na}$ determinação do crescimento das árvores, realizaram-se inventários em todos os tratamentos do experimento, mensurando altura e circunferência a altura do peito (CAP; $1,30 \mathrm{~m}$ ) a partir do 30 mês e a cada três meses, iniciados em março de 2004 e finalizados em janeiro de 2006. A CAP começou a ser mensurada a partir dos 15 meses após o plantio.

$\mathrm{Na}$ determinação da distribuição de raízes finas no perfil de solo, baseado no melhor crescimento das espécies de leguminosas, foram selecionados os tratamentos: T3, E. grandis consorciado com M. scabrella, T6, E. grandis consorciado com M. caesalpiniaefolia e T7, E. grandis consorciado com A. mangium. Na coleta de raízes finas de ambas espécies, foi utilizada uma sonda de $4,5 \mathrm{~cm}$ de diâmetro interno por 1,30 $\mathrm{m}$ de comprimento, inserida no solo até $1 \mathrm{~m}$ de profundidade. A dinâmica de exploração do solo pelas raízes finas do E. grandis e das leguminosas foi estudada de modo a avaliar a capacidade competitiva dessas espécies por água e nutrientes em diferentes camadas do solo e, assim, obter melhor compreensão sobre os fatores que influenciam a produção de biomassa. As raízes finas foram amostradas aos $6(6 / 2004), 16(3 / 2005)$ e 24 meses (1/2006) após o plantio.

Foram escolhidas cinco árvores de E. grandis com diâmetro à altura do peito (DAP) semelhante à média do DAP em cada parcela, ao lado de árvores de leguminosas com crescimento médio em altura e DAP. A partir do tronco de cada espécie leguminosa, coletaramse amostras de solo em cinco pontos diferentes: dois na linha de plantio, às distâncias de 50 e $100 \mathrm{~cm}$, e três na entrelinha de plantio, às distâncias de 50, 100 e $150 \mathrm{~cm}$. Em cada ponto de amostragem, retiraram-se amostras de quatro camadas de solo $(0-10,10-30,30-50$ e $50-100 \mathrm{~cm}$ de profundidade). A fim de não confundir raízes finas das árvores estudadas com raízes de plantas daninhas, estas últimas eram removidas um mês antes da amostragem.

Após a lavagem e limpeza das raízes finas, uma imagem digitalizada das raízes foi produzida por meio de um scanner de mesa. Por meio das imagens das raízes digitalizadas, estimou-se o comprimento radicular com uso do SIARCS (Sistema Integrado para Análise de Raízes e Cobertura do Solo), desenvolvido pela Embrapa Instrumentação Agropecuária (Jorge et al., 1996). As raízes vivas e as mortas de cada espécie foram classificadas visualmente com base nas características morfológicas, coloração, flexibilidade e quantidade de bifurcações.

Raízes de E. grandis foram identificadas por apresentarem maior quantidade de bifurcações do que as raízes das leguminosas e por uma coloração marromclara (Mello, 1997). Raízes de A. mangium e de M. caesalpiniaefolia apresentaram coloração amareloclara, quase translúcida. M. scabrella apresentou raízes mais grossas e coloração avermelhada. Foram amostradas apenas raízes com diâmetro inferior a $3 \mathrm{~mm}$. Raízes de A. mangium e de M. caesalpiniaefolia apresentaram diâmetro médio maior que $1 \mathrm{~mm}$ e menor que $3 \mathrm{~mm}$, respectivamente, e, M. scabrella, entre $2 \mathrm{e}$ $3 \mathrm{~mm}$.

Com a finalidade de determinar a massa de folhas, galhos vivos e mortos, lenho e casca das árvores, foram selecionadas e colhidas seis árvores com DAP médio em cada parcela dos tratamentos 3,5,6 e 7: três árvores de $E$. grandis e três de leguminosas. No tratamento em que o E. grandis foi plantado sozinho (tratamento 5), foram colhidas ao acaso três árvores com DAP médio por parcela. Para determinação da massa seca, foram coletadas folhas, galhos vivos e mortos, que, após homogeneização, foram separadas em amostras de cerca de $100 \mathrm{~g}$ de cada componente. As amostras de lenho foram retiradas de $2 \mathrm{em} 2 \mathrm{~m}$ das árvores cortadas. Separou-se o lenho da casca e anotou-se a massa fresca respectiva de cada árvore. As amostras de folhas, galhos vivos e mortos, lenho e casca foram secadas em estufa $\left(65^{\circ} \mathrm{C}\right)$ até massa constante. Com base nos dados relativos à massa fresca e seca das amostras, bem como à massa fresca total, estimaram-se as massas secas totais destes componentes. As amostras secas e moídas foram analisadas quimicamente para determinação de $\mathrm{N}$ por digestão sulfúrica, conforme o método descrito por Malavolta et al. (1997).

O crescimento relativo do E. grandis foi calculado pela razão entre sua biomassa total nos tratamentos consorciados e sua biomassa total no tratamento com E. grandis puro. A avaliação da FBN foi realizada mediante uso da abundância natural do ${ }^{15} \mathrm{~N}$ nos tecidos de espécies fixadoras de $\mathrm{N}_{2}$ e não-fixadoras, utilizadas como plantas/espécies referência. Essa técnica baseia- 
se no mesmo princípio da técnica de diluição isotópica: a quantidade do $\mathrm{N}$ derivado do ar é proporcional à diferença de enriquecimento de ${ }^{15} \mathrm{~N}$ entre $\mathrm{o} \mathrm{N}$ derivado do solo, estimado pelo enriquecimento da planta controle, e o enriquecimento do $\mathrm{N}$ da fixadora de $\mathrm{N}_{2}$ (Boddey et al., 2000). A fixação biológica de $\mathrm{N}_{2}$ foi estimada comparando-se a abundância natural de ${ }^{15} \mathrm{~N}$ nas leguminosas e no $E$. grandis, espécie não-fixadora de $\mathrm{N}_{2}$. Folhas do terço médio superior de três árvores das leguminosas e três árvores do E. grandis foram amostradas nos tratamentos 3 e 7 , e, no tratamento 5 , folhas do terço médio superior de três árvores de E. grandis. Os três blocos foram amostrados, originando três amostras compostas de cada espécie. Nos tratamentos em que as leguminosas apresentaram baixo crescimento (T1, P. dubium; T2, Inga sp.; T6, M. caesalpiniaefolia), foram amostradas folhas do terço médio superior aleatoriamente nos três blocos. Em média foram amostradas $10 \mathrm{~g}$ de folhas frescas de cada espécie, as quais foram identificadas e secadas em estufa com ventilação forçada $\left(65^{\circ} \mathrm{C}\right)$ até atingir massa constante.

A composição isotópica das amostras foi determinada por um espectrômetro de massa modelo Delta Plus, Thermo Electron, acoplado a um analisador elementar (Carlo Erba NA 1110 CHNS, CE Instruments). A composição isotópica das folhas, expressa como desvio por mil (\%o) em relação a um padrão primário, foi obtida com a equação:

$\delta^{15} \mathrm{~N}=1000 \times\left(\frac{\mathrm{R}_{\text {amostra }}-\mathrm{R}_{\text {padrão }}}{\mathrm{R}_{\text {padrão }}}\right)$

em que $\mathrm{R}_{\text {amostra }}$ é a razão isotópica ${ }^{15} \mathrm{~N} /{ }^{14} \mathrm{~N}$ da amostra, e $\mathrm{R}_{\text {padrão }}$ é a razão isotópica ${ }^{15} \mathrm{~N} /{ }^{14} \mathrm{~N}$ do padrão. Para o $\mathrm{N}$, o padrão internacional é o $\mathrm{N}$ atmosférico $(0,3663 \%)$.

Para estimar a contribuição da fixação biológica do $\mathrm{N}_{2}$ atmosférico (FBN), usou-se a equação, citada por Boddey et al. (2000):

$\% \mathrm{FBN}=\left(\frac{\delta^{15} \mathrm{~N} \text { planta testemunha }-\delta^{15} \mathrm{~N} \text { planta-teste }}{\delta^{15} \mathrm{~N} \text { planta testemunha }-\beta}\right) \times 100$

em que $\delta^{15} \mathrm{~N}$ da planta testemunha é o valor do $\delta^{15} \mathrm{~N}$ do $\mathrm{N}$ absorvido do solo, obtido em folhas de E. grandis, utilizadas como referência não-fixadora; $\delta^{15} \mathrm{~N}$ da planta-teste é o valor de $\delta^{15} \mathrm{~N}$ da planta fixadora de $\mathrm{N}_{2}$; e $\beta$ é o valor da discriminação isotópica de ${ }^{15} \mathrm{~N}$ feita pelas plantas durante o processo de FBN.
O E. grandis foi escolhido como referência nãofixadora por ser uma espécie arbórea, como as leguminosas utilizadas, e por explorar as mesmas camadas de solo o que permite, portanto, o uso dos mesmos estoques de $\mathrm{N}$ mineral no solo. Em trabalho realizado recentemente na Amazônia, Gehring \& Vlek (2004) concluíram que os valores de $\beta$ variaram entre $0 \%$ e $-2 \%$. Neste trabalho foi considerado $\beta$ igual a $0 \%$ para avaliar a FBN das espécies leguminosas.

$\mathrm{Na}$ quantificação da FBN foi considerado que: o comportamento das micorrizas de todas as espécies sobre o $\delta^{15} \mathrm{~N}$ absorvido foi o mesmo, a razão de $\delta^{15} \mathrm{~N}$ nas folhas das espécies leguminosas e no E. grandis foi igual à razão nas árvores inteiras, e o valor $\beta$ é igual a $0 \%$ em todas as espécies. Essas hipóteses, no entanto, nunca podem ser completamente verificadas nas avaliações de FBN em plantações florestais no campo (Boddey et al., 2000; Spriggs et al., 2003). Foram realizadas análises de regressão para determinar o grau de relação entre as variáveis massa e comprimento de raízes finas e a melhor equação que descrevesse a relação entre essas variáveis. Para isto os dados foram analisados no SAS Institute (1996). Todos os gráficos foram confeccionados no programa Sigmaplot (2002).

$\mathrm{Na}$ comparação da densidade de raízes finas entre os tratamentos, os dados foram transformados em $(x+0,5)^{0,5}$, a fim de diminuir a variabilidade entre eles. Em seguida, foi realizada a análise de variância. A biomassa seca de cada compartimento, a concentração de $\mathrm{N} \mathrm{e}$ a análise isotópica de duas espécies (A. mangium e M. scabrella) foram submetidas ao teste de Tukey, a $5 \%$ de probabilidade.

\section{Resultados e Discussão}

Os resultados das medições de crescimento nos consórcios indicam diferentes graus de interações interespecíficas. A altura do $E$. grandis consorciado nos diferentes tratamentos só diferiu do plantio puro de E. grandis aos 24 meses de idade (Figura 1 A). Nesta idade, o E. grandis consorciado com a M. scabrella cresceu menos em altura do que o plantio puro de E. grandis. M. scabrella e A. mangium foram as leguminosas que mais cresceram (Figura $1 \mathrm{~B}$ ), e a primeira chegou a diminuir o crescimento em altura do $E$. grandis. Não houve diferenças no crescimento em área basal do E. grandis nos tratamentos consorciados com as leguminosas de maior crescimento, em relação ao E. grandis puro (Figura $1 \mathrm{C}$ e D). 
O P. dubium, o Inga sp. e a A. polyphylla apresentaram crescimento muito baixo. Com menos de 6 meses de idade, essas espécies haviam sido superadas em altura pelo E. grandis (Figura $1 \mathrm{~B}$ ). As espécies mais competitivas, A. mangium e M. scabrella, só foram sobrepujadas em altura pelo E. grandis depois dos 12 meses.

Quando se considera os compartimentos da planta separadamente, houve diferença apenas quanto à produção de biomassa de lenho de E. grandis solteiro em relação ao consorciado com A. mangium. Quanto à soma da biomassa de folha, galho e casca, não houve diferenças entre tratamentos com E. grandis solteiro ou consorciado. Entretanto, quando se considera a biomassa total, o E. grandis solteiro produziu mais do que no tratamento em que foi consorciado com A. mangium; nos demais tratamentos essa diferença não foi estatisticamente significativa (Tabela 1). Considerando-se o crescimento relativo em biomassa total de E. grandis consorciado com M. scabrella $(0,83)$, com M. caesalpiniaefolia $(0,93)$ e com A. mangium $(0,79)$, em relação a $E$. grandis puro $(1,00)$, constatase que a competição interespecífica foi maior que a intraespecífica, pelo menos até os 24 meses de idade. Apesar de ter ocorrido redução na produção do eucalipto, o consórcio com $M$. scabrella revela maior acumulação de biomassa (Tabela 1). A espécie com maior biomassa de copa (folha + galho) aos dois anos foi A. mangium (Tabela 1). Na avaliação dos valores totais de cada
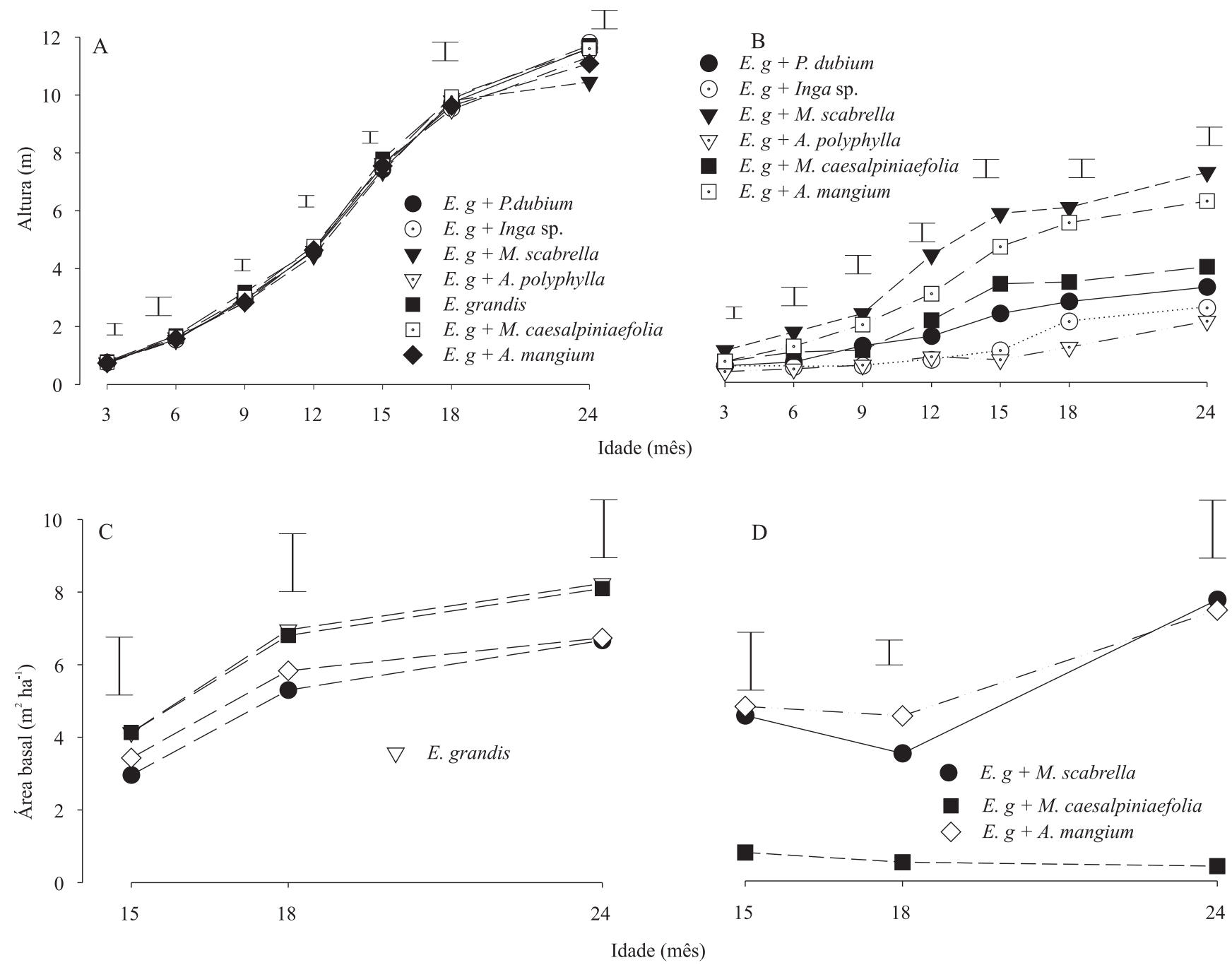

Figura 1. Crescimento em altura dos 3 aos 24 meses pós-plantio do E. grandis (E.g.) (A) e das leguminosas consorciadas (B); crescimento em área basal dos 15 aos 24 meses pós-plantio do E. grandis (C) e da A. manguim, M. scabrella e M. caesalpiniaefolia (D). As barras representam a diferença mínima significativa pelo teste de Tukey, a 5\% de probabilidade. 
consórcio, somente o tratamento 3 mostrou-se mais produtivo do que o plantio puro.

Apesar da menor massa de folhas aos dois anos, M. scabrella produziu mais lenho do que A. mangium. Esse resultado indica maior eficiência de produção de lenho pela $M$. scabrella, o que está relacionado ao menor dispêndio de fotoassimilados para síntese de galhos. Esta espécie ocorre naturalmente em povoamentos densos na Região Sul do Brasil, com alta população, em média de 1.400 a 3.000 indivíduos por hectare (Machado et al., 2001). Esse comportamento evidencia alta capacidade de competição interespecífica, em parte explicado pela eficiência fotossintética das copas e pela alocação preferencial de fotoassimilados no lenho (Inoue \& Galvão, 1986). Esta eficiência fotossintética está relacionada com a estrutura de copas de M. scabrella em povoamentos mistos. Suas folhas são muito pequenas e concentradas nas extremidades dos galhos, o que confere à espécie maior flexibilidade na busca por espaço luminoso, e diminui o sombreamento interespecífico.

A maior produção de folhas de $A$. mangium fez com que ela competisse mais por água e luz com $E$. grandis do que $M$. scabrella (Figura 1 B), que produziu menos folhas e apresentou estrutura de copas mais favorável à competição interespecífica. Assim, M. scabrella foi mais eficiente que A. mangium na produção de lenho. A produção total de lenho do consórcio E. grandis e M. scabrella foi de $21,9 \mathrm{Mg} \mathrm{ha}^{-1}$; superior a $E$. grandis puro, aos 24 meses de idade (Tabela 1).

A maior variação da densidade de raízes finas (DRF) de E. grandis ocorreu na camada de $0-10 \mathrm{~cm}$ de profundidade, nos diferentes tratamentos. Abaixo dessa camada, a DRF diminuiu fortemente (Figura 2). Na camada de solo 0-30 cm, Mello (2004) encontrou mais de $80 \%$ do comprimento total das raízes finas amostradas até $1 \mathrm{~m}$ de profundidade. Segundo Gonçalves et al. (2001) e Laclau et al. (2004), este efeito se deve à maior concentração de nutrientes na camada superficial, enriquecida pela ciclagem biogeoquímica de nutrientes. Diminuição rápida das concentrações de nutrientes nas soluções foi observada nas camadas superficiais do solo em plantações de Eucalyptus no Congo (Laclau et al., 2004) e no Brasil (Maquère et al., 2005). A predominância das raízes de E. grandis na camada $0-30 \mathrm{~cm}$ indica vantagem competitiva dessa espécie sobre as leguminosas no acesso aos nutrientes e à água infiltrada no solo após chuvas de baixa intensidade. Por sua vez, a DRF de

Tabela 1. Biomassa seca e concentração de $\mathrm{N}$ em diferentes compartimentos da planta aos 2 anos de idade ${ }^{(1)}$.

\begin{tabular}{|c|c|c|c|c|c|c|}
\hline Tratamento & Folha & Galho morto & Galho vivo & Lenho & Casca & Biomassa total \\
\hline & \multicolumn{6}{|c|}{ Biomassa seca $\left(\mathrm{Mg} \mathrm{ha}^{-1}\right)$} \\
\hline E. grandis + M. scabrella & $4,1 \mathrm{a}$ & $4,7 \mathrm{a}$ & $1,5 \mathrm{a}$ & $14,4 \mathrm{ab}$ & $3,0 \mathrm{a}$ & $27,7 \mathrm{ab}$ \\
\hline E. grandis puro & $4,6 \mathrm{a}$ & $5,5 \mathrm{a}$ & $2,1 \mathrm{a}$ & $17,6 \mathrm{~b}$ & $3,3 \mathrm{a}$ & $33,1 b$ \\
\hline E. grandis $+M$. caesalpiniaefolia & $3,7 \mathrm{a}$ & $5,4 \mathrm{a}$ & $2,4 \mathrm{a}$ & $16,3 \mathrm{ab}$ & $3,4 \mathrm{a}$ & $31,1 \mathrm{ab}$ \\
\hline E. grandis $+M$. mangium & $3,4 \mathrm{a}$ & $4,5 \mathrm{a}$ & $1,8 \mathrm{a}$ & $13,6 \mathrm{a}$ & $2,9 \mathrm{a}$ & $26,2 a$ \\
\hline & \multicolumn{6}{|c|}{ Leguminosa } \\
\hline E. grandis + M. scabrella & $1,0 \mathrm{ab}$ & $1,3 a$ & $1,1 b$ & $7,5 b$ & $1,2 b$ & $12,1 \mathrm{~b}$ \\
\hline E. grandis + M. caesalpiniaefolia & $0,1 \mathrm{a}$ & $0,1 \mathrm{a}$ & $0,1 \mathrm{a}$ & $1,0 \mathrm{a}$ & $0,1 \mathrm{a}$ & $1,6 \mathrm{a}$ \\
\hline E. grandis $+M$. mangium & $2,1 \mathrm{~b}$ & $2,1 b$ & $0,1 \mathrm{a}$ & $2,6 \mathrm{a}$ & $1,0 \mathrm{ab}$ & $7,2 \mathrm{ab}$ \\
\hline \multicolumn{7}{|c|}{ Total (E. grandis + leguminosa) } \\
\hline E. grandis + M. scabrella & $5,1 b$ & $6,0 \mathrm{ab}$ & $2,6 \mathrm{~b}$ & $21,9 b$ & $4,2 b$ & $39,8 b$ \\
\hline E. grandis puro & $4,6 b$ & $5,5 \mathrm{a}$ & $2,1 \mathrm{ab}$ & $17,6 \mathrm{a}$ & $3,3 \mathrm{a}$ & $33,1 \mathrm{a}$ \\
\hline E. grandis + M. caesalpiniaefolia & $3,8 \mathrm{a}$ & $5,5 \mathrm{a}$ & $2,5 \mathrm{ab}$ & $17,3 \mathrm{a}$ & $3,5 \mathrm{a}$ & $32,7 a$ \\
\hline E. grandis $+M$. mangium & $5,5 \mathrm{~b}$ & $6,6 \mathrm{~b}$ & $1,9 \mathrm{a}$ & $16,2 \mathrm{a}$ & $3,9 \mathrm{ab}$ & $33,4 a$ \\
\hline & \multicolumn{6}{|c|}{$\begin{array}{c}\text { Concentração de } \mathbf{N}\left(\mathbf{g ~ k g}^{-1}\right) \\
\text { Eucalyptus grandis }\end{array}$} \\
\hline E. grandis + M. scabrella & $16,4 \mathrm{a}$ & - & $3,7 \mathrm{a}$ & $3,2 \mathrm{a}$ & $1,8 \mathrm{a}$ & - \\
\hline E. grandis puro & $17,7 \mathrm{a}$ & - & $4,3 \mathrm{a}$ & $2,9 \mathrm{a}$ & $1,6 \mathrm{a}$ & - \\
\hline E. grandis + M. caesalpiniaefolia & $17,3 \mathrm{a}$ & - & $3,7 \mathrm{a}$ & $3,3 \mathrm{a}$ & $1,6 \mathrm{a}$ & - \\
\hline E. grandis $+M$. mangium & $18,1 \mathrm{a}$ & - & $3,5 \mathrm{a}$ & $3,3 \mathrm{a}$ & $1,6 \mathrm{a}$ & - \\
\hline & \multicolumn{6}{|c|}{ Leguminosa } \\
\hline E. grandis + M. scabrella & $35,0 \mathrm{c}$ & - & $13,0 \mathrm{~b}$ & $18,7 \mathrm{c}$ & $3,2 b$ & - \\
\hline E. grandis $+M$. caesalpiniaefolia & $29,5 b$ & - & $5,2 \mathrm{a}$ & $13,7 b$ & $3,0 \mathrm{ab}$ & - \\
\hline E. grandis $+A$. mangium & $21,1 \mathrm{a}$ & - & $5,9 \mathrm{a}$ & $9,1 \mathrm{a}$ & $2,5 \mathrm{a}$ & - \\
\hline
\end{tabular}

${ }^{(1)}$ Médias seguidas pela mesma letra não diferem entre si pelo teste de Tukey, a $5 \%$ de probabilidade. 


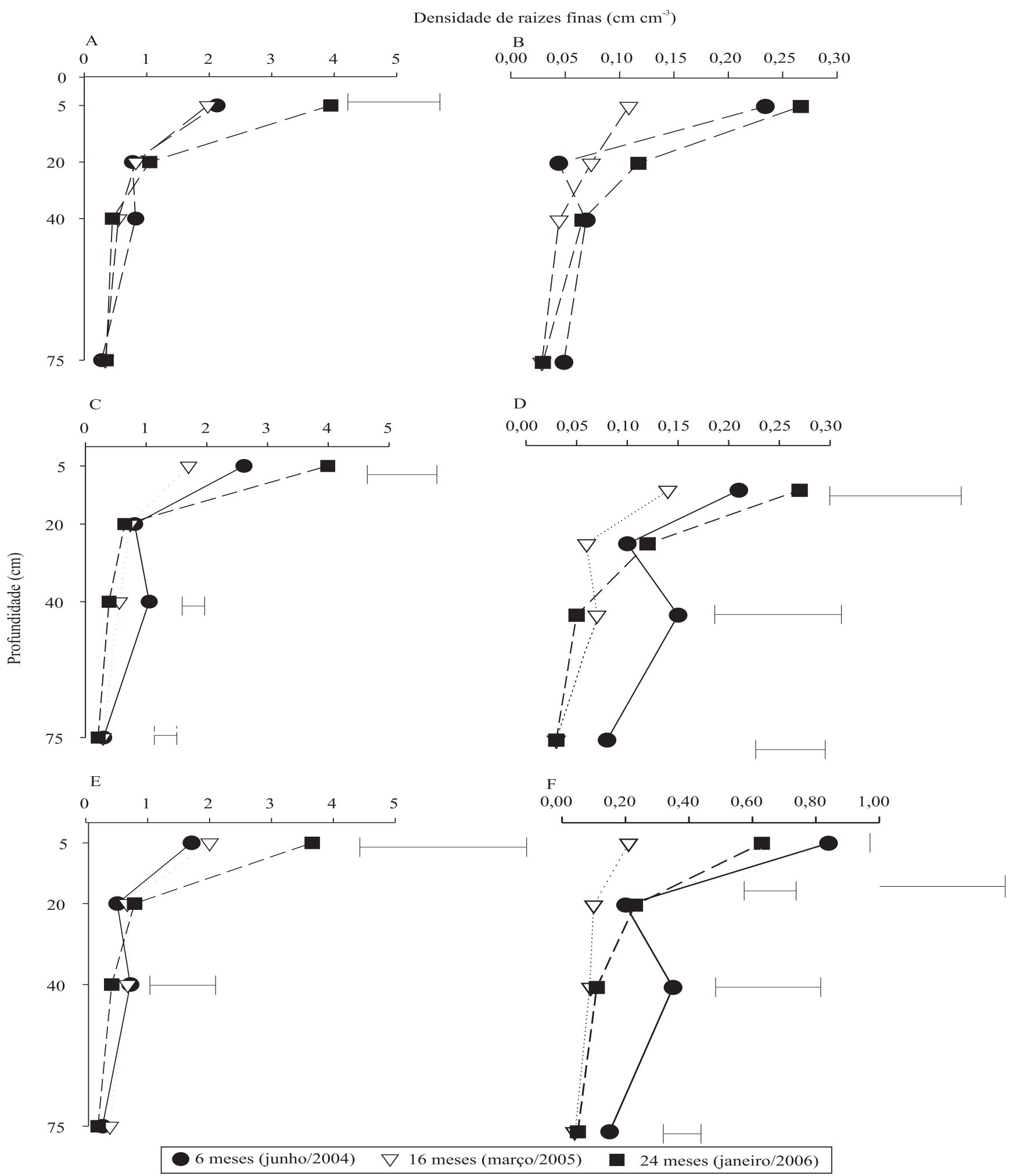

Figura 2. Densidade média de raízes finas de E. grandis (A, C e E) e de A. mangium, M. caesalpiniaefolia e M. scabrella (B, D e F, respectivamente) em plantio consorciado com diferentes idades pós-plantio. As profundidades apresentadas representam pontos intermediários das profundidades amostradas $(0-10 ; 10-30 ; 30-50 ; 50-100 \mathrm{~cm})$. As barras representam a diferença mínima significativa pelo teste de Tukey, a $5 \%$ de probabilidade. 
M. caesalpinieafolia e M. scrabella aumentou na camada $30-50 \mathrm{~cm}$ em relação à camada $10-30 \mathrm{~cm}$. Nessa última, observaram-se maiores DRF até na camada mais profunda de amostragem $(50-100 \mathrm{~cm})$, sugerindo maior eficiência na aquisição de nutrientes nas camadas mais profundas. Em todas as espécies, houve crescimento de raízes entre 16 e 24 meses de idade. Em plantios de Leucena leucocephala consorciada com milho e ervilha, Akinnifesi et al. (1995) verificaram que, em dez anos de consórcio, 40\% do total de raízes finas da L. leucocephala ocorreram em profundidades maiores que $60 \mathrm{~cm}$, enquanto $98 \%$ do total de raízes do milho e da ervilha foram encontradas na camada entre 0 e $60 \mathrm{~cm}$. Esse tipo de comportamento é um dos recursos relativos à plasticidade do sistema radicular em resposta às interações interespecíficas para acesso à água e nutrientes no solo.

A. mangium e $M$. caesalpinieafolia apresentaram as menores DRF (menor que $0,30 \mathrm{~cm} \mathrm{~cm}^{-3}$ ) em todas as camadas de solo até $1 \mathrm{~m}$ de profundidade. M. scrabella foi a espécie com a maior DRF, a qual chegou a $0,84 \mathrm{~cm} \mathrm{~cm}^{-3}$ na camada $0-10 \mathrm{~cm}$ aos seis meses (Figura 2). Apesar de apresentar menor biomassa de raízes finas, $M$. scabrella conseguiu competir em altura até 12 meses de idade com E. grandis. A DRF de todas as leguminosas foi sempre menor do que a de E. grandis, que, na camada superior, em quase todas as amostragens, apresentou DRF maior que $2 \mathrm{~cm} \mathrm{~cm}^{-3}$, o que indica forte competição interespecífica nessa camada. Estes valores são aproximadamente $30 \%$ maiores que os encontrados num povoamento puro de E. globulus aos seis anos de idade (Bauhus et al., 2000). Esta diferença provavelmente ocorreu em virtude da maior produtividade de E. grandis observada.

As concentrações de $\mathrm{N}$ encontradas nas folhas de E. grandis não foram modificadas pelas espécies leguminosas consorciadas, o que indica que a transferência de $\mathrm{N}$ das leguminosas para $E$. grandis foi baixa ou inexistente até a idade de dois anos (Tabela 1). O mesmo resultado foi encontrado por Bouillet et al. (2006), em plantações consorciadas de E. grandis e A. mangium aos 2,5 anos de idade. Por sua vez, aumento das concentrações de $\mathrm{N}$ nas raízes finas de E. globulus, nesta idade, foi verificado por Khanna (1997) em consórcios com Acacia mearnsii.
As concentrações foliares de $\mathrm{N} \mathrm{em} E$. grandis encontramse dentro da faixa de normalidade definida por Silveira et al. (2000). O mesmo ocorre com relação a $M$. scabrella, segundo Baggio \& Carpanezzi (1997), e a A. mangium (Garay et al., 2004) (Tabela 1). Em todos os tratamentos, apenas o consórcio entre $E$. grandis e $M$. scabrella apresentou maior acúmulo de $\mathrm{N}$ na soma de todos os seus compartimentos em relação ao povoamento solteiro de E. grandis (Figura 3). A M. scabrella foi mais eficiente do que as demais leguminosas em incorporar $\mathrm{N}$ na biomassa aérea. Além de maior produção de biomassa, as concentrações de $\mathrm{N}$ foram maiores em todos os compartimentos aéreos nessa espécie (Tabela 1).

Os valores de $\delta^{15} \mathrm{~N}$ foram menores que $1 \%$ o nas folhas de M. scabrella e Inga sp., e maiores que 5\%o nas folhas de P. dubium e A. mangium. Ainda que o número de amostras seja limitado, a grande amplitude de $\delta^{15} \mathrm{~N}$ constitui indicação de que a fixação biológica de $\mathrm{N}$ atmosférico, por M. scabrella e Inga sp., foi maior que a fixação por P. dubium e A. mangium. A elevada FBN por M. scabrella, indicada pela determinação de $\delta^{15} \mathrm{~N}$ em amostras coletadas em três blocos experimentais, está de acordo com as concentrações altas de $\mathrm{N}$ encontradas nas folhas dessa espécie. Essa informação sobre a FBN de espécies nativas pouco estudadas é importante a fim de avaliar seus potenciais de fixação de $\mathrm{N}$ em plantações consorciadas.

A quantidade de $\mathrm{N}$ derivado daFBN, estimada pelo método da abundância natural de ${ }^{15} \mathrm{~N}$, foi de $92 \%$ em M. scabrella e 74\% em Inga sp. e M. caesalpiniaefolia, apesar do baixo desenvolvimento dessas últimas espécies. Contudo, considerando que todos os requisitos necessários à aplicação desse método de avaliação por abundância natural foram satisfeitos, a quantificação da FBN foi pouco precisa. Os valores de $\delta^{15} \mathrm{~N}$, em algumas leguminosas, mais altos do que na espécie-referência, não-fixadora, evidenciam as limitações do método. Os baixos valores de $\delta^{15} \mathrm{~N}$ nas folhas de $E$. grandis desaconselham a aplicação do método de abundância natural de $\mathrm{N}$ para quantificar a FBN.

Segundo a recomendação de Högberg (1997), o $\delta^{15} \mathrm{~N}$ da espécie-referência deve ser no mínimo 5\%o maior que o valor $\beta$ para estimar a fixação biológica de $\mathrm{N}$, o que neste trabalho não foi satisfeito. Apesar dessa restrição, as amplas diferenças entre as leguminosas constituem indicativo útil sobre o potencial de $M$. scabrella para aumentar a disponibilidade de $\mathrm{N}$ no solo em plantações consorciadas. 


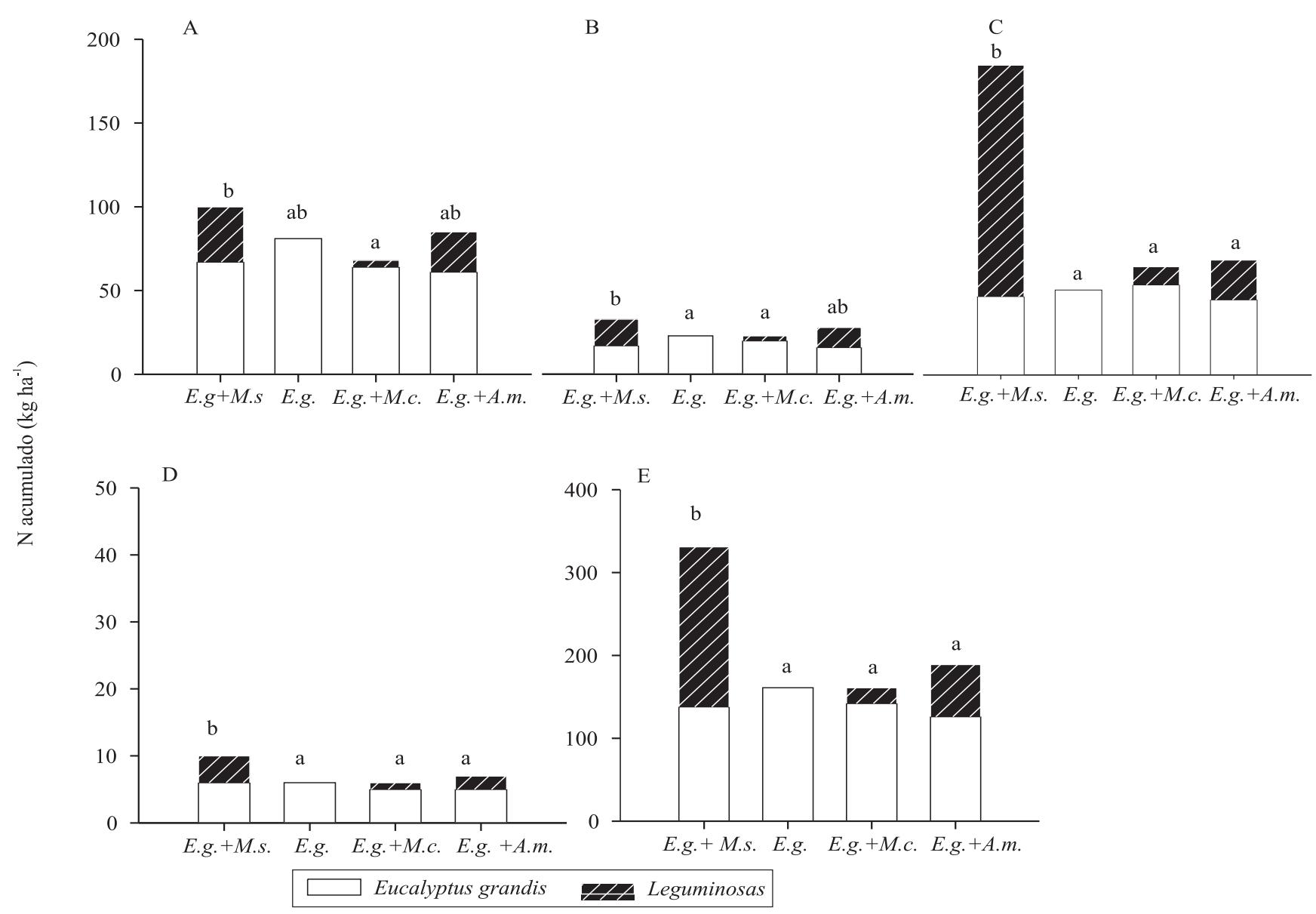

Figura 3. Nitrogênio acumulado na folha (A), no galho vivo (B), no lenho (C), na casca (D) e em todos os compartimentos (E) nos diferentes tratamentos aos 2 anos de idade. Médias com letras iguais não diferem entre si pelo teste de Tukey, a 5\% de probabilidade. E.g., M.s., M.c. e A.m. são, respectivamente, Eucalyptus grandis, Mimosa scabrella, Mimosa caesalpiniaefolia e Acacia mangium.

\section{Conclusões}

1. A competição interespecífica entre árvores de E. grandis e leguminosas é maior do que a intraespecífica até a idade de 24 meses.

2. Entre as leguminosas estudadas, A. mangium e M. scabrella foram as que melhor resistiram à competição com o E. grandis.

3. A distribuição do sistema radicular de E. grandis, de $M$. scabrella e de A. mangium no perfil do solo indica que existem nichos de exploração radicular diferentes entre as espécies.

4. M. scabrella é mais eficiente quanto ao acúmulo de $\mathrm{N}$ na biomassa da parte aérea até a idade de 24 meses.

\section{Referências}

AKINNIFESI, F.K.; KANG, B.T.; TIJANI-ENIOLA, H. Root size distribution of a Leucaena leucocephala hedgerows as effected by pruning and alley cropping. Nitrogen Fixing Trees Research Report, v.13, p.65-69, 1995.

BAGGIO, A.J.; CARPANEZZI, A.A. Exportação de bracatingais. Boletim de Pesquisa Florestal, v.34, p.3-15, 1997.

BALIEIRO, F.C.; FONTES, R.L.F.; DIAS, L.E.; FRANCO, A.A.; CAMPELLO, E.F.C.; FARIA, S.M. de. Accumulation and distribution of aboveground biomass and nutrients in pure and mixed stands of guachapele and eucalyptus. Journal of Plant Nutrition, v.25, p.2639-2654, 2002.

BARROS, N.F.; NOVAIS, R.F.; NEVES, J.C.L. Fertilização e correção do solo para o plantio de eucalipto. In: BARROS, N.F.; NOVAIS, R.F. (Ed.). Relação solo-eucalipto. Viçosa: Ed. Viçosa, 1990. p.127-186. 
BAUHUS, J.; KHANNA, P.K.; MENDEN, N. Aboveground and belowground interactions in mixed plantations of Eucalyptus globulus and Acacia mearnsii. Canadian Journal of Forest Research, v.30, p.1886-1894, 2000.

BINKLEY, D.; GIANDINA, C.; BASHKIN, M.A. Soil phosphorous pools and supply under the influence of Eucalyptus saligna and nitrogen fixing Albizia facaltaria. Forest Ecology and Management, v.128, p.241-247, 2000.

BODDEY, R.M.; PEOPLES, M.B.; PALMER, B.; DART, P.J. Use of ${ }^{15} \mathrm{~N}$ natural abundance technique to quantify biological nitrogen fixation by woody perennials. Nutrient Cycling in Agroecosystems, v.57, p.235-270, 2000.

BOUILLET, J.P.; LACLAU, J.P.; GONÇALVES, J.L.M.; MOREIRA, M.Z.; TRIVELIN, P.; JOURDAN, C.; GALIANA, A. Mixed-species plantations of Acacia mangium and Eucalyptus grandis in Brazil. In: WORKSHOP ON SITE MANAGEMENT AND PRODUCTIVITY IN TROPICAL PLANTATION FORESTS, 7., Bogor. Proceedings. Bogor: Center for International Forestry Research, 2006. p.1-13.

FORRESTER, D.I.; BAUHUS, J.; COWIE, A.L.; VANCLAY, J.K. Mixed-species plantations of Eucalyptus with nitrogen-fixing trees: a review. Forest Ecology and Management, v.233, p.211-230, 2006.

GARAY, I.; PELLENS, R.; KINDEL, A.; BARROS, E.; FRANCO, A. Evaluation of soil conditions in fast-growing plantations of Eucalyptus grandis and Acacia mangium in Brazil: a contribution to the study of sustainable land use. Applied Soil Ecology, v.27, p.177187, 2004.

GEHRING, C.; VLEK, P.L.G. Limitations of the ${ }^{15} \mathrm{~N}$ natural abundance method for estimating biological nitrogen fixation in Amazonian forest legumes. Basic and Applied Ecology, v.5, p.567580, 2004.

GONÇALVES, J.L.M.; MENDES, K.C.F.S.; SASAKI, C.M. Mineralização de nitrogênio em ecossistemas florestais naturais implantados do Estado de São Paulo. Revista Brasileira de Ciência do Solo, v.25, p.601-616, 2001.

HÖGBERG, P. ${ }^{15} \mathrm{~N}$ natural abundance as a possible marker of the ectomycorrhizal habit of trees in mixed African woodlands. New Phytologist, v.115, p.483-486, 1997.

INOUE, T.M.; GALVÃO, F. Desempenho assimilatório de Mimosa scabrella, Peltrphorum dubium, Schinus terebinthifolius e Natayba elacagnoides em dependência da intensidade luminosa. Acta Forestalia Brasiliensis, v.1, p.89-98, 1986.

JORGE, L.A.C.; RALISCH, R.; ABI SAAB, O.J.G.; MEDINA, C.C.; GUIMARÃES, M.F.; NEVES, C.S.V.J.; CRESTANA, S.; CINTRA, F.L.D.; BASSOI, L.H.; FERNANDES, S.B.V. Aquisição de imagens de raízes. In: JORGE, L.A.C. (Ed.). Recomendações práticas para aquisição de imagens digitais através do SIARCS. São Carlos: Embrapa-CNPDIA, 1996. 28p. (Circular técnica, 1).

KHANNA, P.K. Comparison of growth and nutrition of young monocultures and mixed stand of Eucalyptus globulus and Acacia mearnsii. Forest Ecology and Management, v.94, p.105-113, 1997.
LACLAU, J.P.; TOUSTAIN, F.; M'BOU, A.T.; ARNAUD, M.; JOFFRE, R.; RANGER, J. The function of the superficial root mat in the biogeochemical cycles of nutrients in Congolenses Eucalyptus plantations. Annals of Botany, v.53, p.249-261, 2004.

LI, Z.; PENG, S.L.; RAE, D.J.; ZHOU, G. Litter decomposition and nitrogen mineralization of soils in subtropical plantation forests of southern China, with special attention to comparisons between legumes and non-legumes. Plant and Soil, v.229, p.105-116, 2001.

MACHADO, S. do A.; TONON, A.E.N.; OLIVEIRA, E.B. de; FIGUEIREDO FILHO, A.; CARPANEZZI, A.A. Efeito da densidade inicial e do sítio sobre o desenvolvimento de bracatingais nativos da região metropolitana de Curitiba-PR. Boletim Pesquisa Florestal, v.43, p.19-46, 2001.

MALAVOLTA, E.; VITTI, G.C.; OLIVEIRA, S.K.L.A. Princípios, métodos e técnicas de avaliação do estado nutricional. In: MALAVOLTA, E.; VITTI, G.C.; OLIVEIRA, S.A. de. Avaliação do estado nutricional das plantas: princípios e aplicações. 2.ed. Piracicaba: Potafos, 1997. 467p.

MAQUÈRE, V.; LACLAU, J.P.; GONÇALVES, J.L.M.; PICCOLO, M.C.; KRUSHE, A.V.; RANGER, J. Influence of fertilizer inputs on the chemistry of soil solutions in eucalypt plantations established on Brazilian sandy soils. In: WORKSHOP ON MANAGEMENT OF TROPICAL SANDY SOIL FOR SUSTAINABLE AGRICULTURE, 27., 2005, Khon Kaen. A holisitic approach for sustainable development of problem soils in the tropics: proceedings. Khon Kaen: International Union of Soil Science, 2005. p.1-9.

MELLO, S.L.M. Características do sistema radicular em povoamentos de eucaliptos propagados por sementes e estacas. 1997. 79p. Dissertação (Mestrado) - Escola Superior de Agricultura Luiz de Queiroz, Piracicaba.

MELLO, S.L.M. Dinâmica de raízes finas e aporte de carbono no solo sob povoamentos de Eucalyptus grandis no Estado de São Paulo. 2004. 84p. Tese (Doutorado) - Universidade de São Paulo, Piracicaba.

O'CONNELL, A.M.; RANCE, S.J. Predicting nitrogen supply in plantation eucalypt forest. Soil Biology and Biochemistry, v.31, p.1943-1951, 1999.

RAIJ, B. van; ANDRADE, H.; CANTARELLA, H.; QUAGGIO, J.A. Análise química para avaliação da fertilidade de solos tropicais. Campinas: Instituto Agronômico, 2001. 285p.

SAS INSTITUTE (Cary, Estados Unidos). SAS/STAT: software and enhancement. Release 6.11. Cary, 1996. 2018p.

SIGMAPLOT software. Version 8. Chicago: SPSS, 2002. 4 CD-ROM.

SILVEIRA, R.L.V.A.; HIGASHI, E.N.; GONÇALVES, A.N.; MOREIRA, A. Avaliação do estado nutricional do Eucalyptus: diagnose visual, foliar e suas interpretações. In: GONÇALVES, J.L.M.; BENEDETTI, V. (Ed.). Nutrição e fertilidade florestal. Piracicaba: Ipef, 2000. p.79-104.

SPRIGGS, A.M.; STOCK, W.D.; DAKORA, F.D. Influence of mycorrhizal association on foliar $\delta^{15} \mathrm{~N}$ values of legume and non legume shrubs and trees in the fynbos of South Africa: implications for estimating $\mathrm{N}_{2}$ fixation using the ${ }^{15} \mathrm{~N}$ natural abundance method. Plant and Soil, v.255, p.495-502, 2003. 